\title{
Copper-Containing Rod-Shaped Nanosized Silica Particles for Microwave-Assisted Synthesis of Triazoles in Aqueous Solution
}

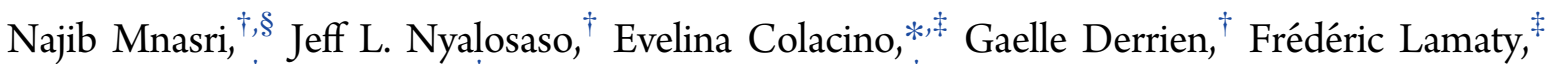 \\ Jean Martinez, ${ }^{\ddagger}$ Jerzy Zajac, ${ }^{\dagger}$ and Clarence Charnay ${ }^{*}{ }^{\dagger}$ \\ ${ }^{\dagger}$ Institut Charles Gerhardt de Montpellier (ICGM), CNRS UMR 5253, Université Montpellier, Campus Triolet, Place Eugène \\ Bataillon, 34095 Montpellier Cedex 5, France \\ ${ }^{*}$ Institut des Biomolécules Max Mousseron (IBMM), UMR 5247 CNRS - UM - ENSCM, Université Montpellier, Campus Triolet, \\ Place E. Bataillon, 34095 Montpellier Cedex 5, France \\ ${ }^{\S}$ Materials, Environment and Energy Laboratory (UR14ES26), Faculty of Science, University of Gafsa, 2112 Gafsa, Tunisia
}

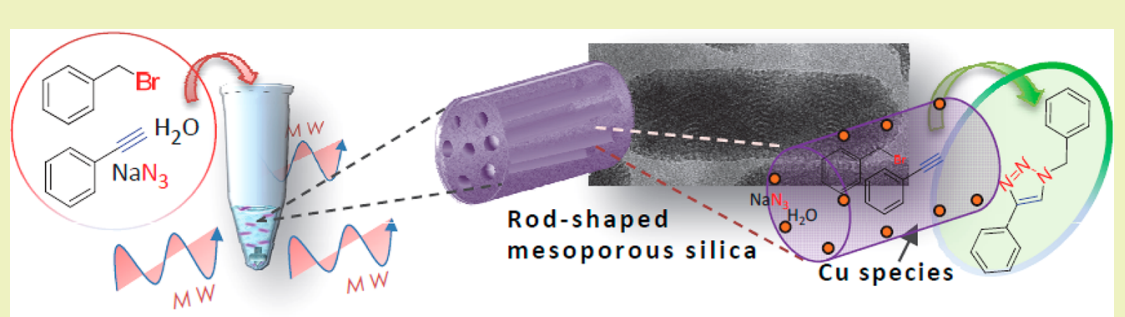

ABSTRACT: $\mathrm{Cu}$-doped rod-shaped mesoporous silica nanoparticles (Cu-RMSN) were synthesized by a new one-step approach based on in situ functionalization procedure referring to the phenomenon of micellar solubilization. SEM and TEM studies revealed the rod-like morphology of uniformly sized particles with longitudinal mesopore channels. The BET specific surface areas were near $500 \mathrm{~m}^{2} \mathrm{~g}^{-1}$ and the average pore diameter varied from 3 to $3.6 \mathrm{~nm}$. The composite Cu-RMSN proved to be an efficient heterogeneous catalyst for a microwave-assisted three-component 1,3-dipolar cycloaddition reaction in aqueous solution. The one-pot preparation of 1,4-dibustituted-1,2,3-triazole derivatives was straightforward and high yielding, due to the high copper dispersion at the pore surface resulting in the high accessibility of the active sites. The efficiency of the catalyst was also demonstrated upon recycling, making such synthesis a truly green process with marked step and solvent economy and important waste reduction.

KEYWORDS: Heterogeneous catalysis, Multicomponent reactions (MCR), Click chemistry, Microwaves, Mesoporous silica, Nanoparticles, Micellar solubilization

\section{INTRODUCTION}

The design of nanoporous materials with the simultaneous control of the particle size, morphology and porosity remains one of the key elements in the context of heterogeneous catalysis applications in solution. ${ }^{1,2}$ Indeed, the resistance to mass transfer of solutes is often large enough to impede the accessibility to the surface reactive sites occurring by a diffusion process at the solid-surface interface. ${ }^{3}$ Mesoporous silica nanoparticles (MSN) display great potential advantages in liquid-phase catalytic processes, because particle size and shape can noticeably modify the transport of the solutes from the bulk solution to the internal pore surface. It is thus expected that morphology be of great importance in the development of more efficient heterogeneous catalysts. $^{4-6}$ Although, rodshaped mesoporous silica nanoparticles maintaining a lengthwise alignment of uniform pore channels are of high current interest in the context of their potential applications in catalysis, ${ }^{1,7,8}$ separation, ${ }^{9}$ sensoring, ${ }^{10,11}$ and nanomedicine, ${ }^{12-16}$ there are only few reports investigating the catalytic efficiency of rod- shaped MSN as supports of active catalytic centers. $^{1,2,8,17}$ It was recently reported that accessibility to acid sites in Al-mesoporous silica nanoparticles could be correlated with the particle size rather than with their morphology. ${ }^{8}$ Compared to mesoporous silica spheres of several hundred nanometers, rod-shaped MSN possessed in fact diameters below hundred nanometers, which resulted in a high accessibly to the active sites.

Moreover, another key focus of MSN applications as catalysts was the introduction of either chemical functionalities or heteroatoms into the silica framework to improve the weak reactivity of silica surfaces. ${ }^{18-22}$ Among the various strategies for the synthesis of small mesoporous silica particles, ${ }^{23}$ the preparation of nanosized mesoporous silica spheres with a controlled size and morphology, as well as regular pore ordering includes the use of base-mediated conditions (i.e., modified Stöber route), ${ }^{24,25}$ which are not compatible with 
Scheme 1. Synthesis Pathway of Metal-Rod Shaped Mesoporous Silica Nanoparticles

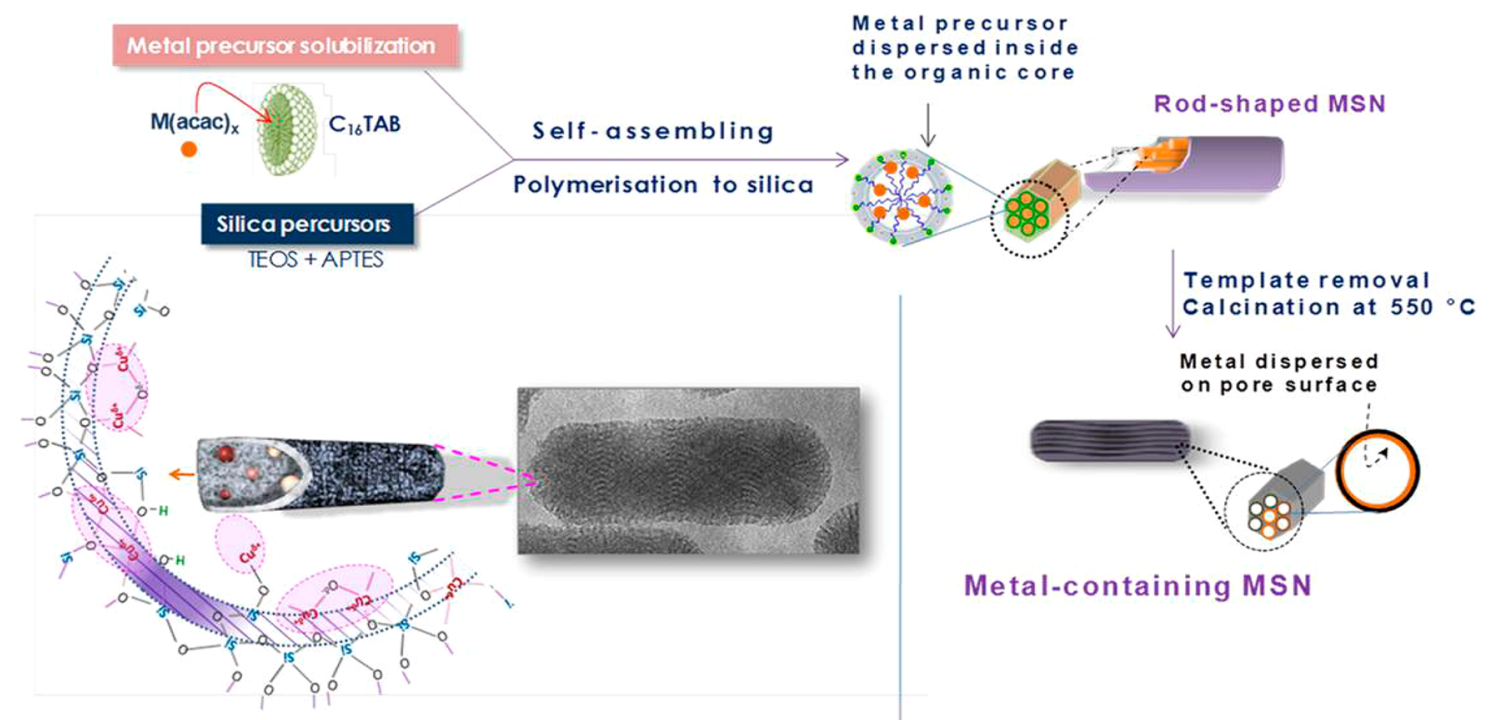

common in situ incorporation of ionic metal precursors. Transition metal inclusion in the MSN framework can be also achieved by a postsynthesis impregnation; nevertheless, the backfilling approach risks clogging of the matrix pores, thereby reducing the pore accessibility. In view of the one-step approach strategy, a new in situ functionalization procedure has been developed in our institute based on the phenomenon of micellar solubilization. ${ }^{26,27}$ This combined synthesis and functionalization approach, depicted in Scheme 1, was for the first time, to the best of our knowledge, adapted for preparing copper-containing rod-shaped MSN with perfect conservation of pore-ordering, shape and monodispersity, and high intrapore metal dispersion. For this purpose, templating micelles of a cationic surfactant in situ loaded with a given amount of copper precursor were expected to play a double role of "soft porogens" to create nanoscopic pore structure and precursordelivery "capsules" for targeted functionalization of the future internal surfaces. Location of the copper precursor inside the micelles was conceived in a way to preclude the metal species from being inserted into the bulk of the solid during silica condensation.

It is well-known that heterogeneous catalysts supporting copper species may regioselectively catalyze the Huisgen 1,3dipolar cycloaddition of (organic) azides and terminal alkynes $(\mathrm{CuAAC})^{28-33}$ in pure solvents ${ }^{34,35}$ or solvent mixtures ${ }^{36,37}$ to achieve 1,2,3-triazole derivatives, an important class of bioactive compounds with, among others, antiallergenic, ${ }^{38}$ antibacteri$\mathrm{al}^{39-41}$ or anti-HIV ${ }^{42,43}$ activities. In addition, immobilized copper has proven to be very efficient in catalyzing the threecomponent reactions of organic halides, sodium azide, and terminal alkynes, allowing the one-pot synthesis of triazoles. $^{44-50}$ This reaction pathway has the advantage of eliminating the use and handling of explosive and hazardous organic azides, as they are generated in situ via a substitution mechanism usually catalyzed by a metallic Lewis acid.

The present paper reports on the one-pot synthesis of $\mathrm{Cu}$ doped rod-shaped mesoporous silica nanoparticles $(\mathrm{Cu}-$ RMSN) with active sites located at the internal surface and thus easily accessible by the reactants, as required for specific surface reactivity in the liquid phase. The classical characterization methods were employed to determine the particle size distribution, textural properties, degree of $\mathrm{Cu}$ incorporation into the silica framework, and accessibility to the active sites in the resulting materials. An eco-friendly microwave-assisted multicomponent reaction in water was subsequently tested, without addition of any other cosolvent or ligand. This "green" approach succeeded in copper-mediated cycloaddition of alkynes, benzyl or alkyl halides, and sodium azide making use of Cu-RMSN as a heterogeneous and recyclable catalyst in aqueous solution, for the first time.

\section{MATERIALS AND METHODS}

All reagents were commercially available and used without any further purification.

Materials Characterizations. Scanning electron microscopy (SEM) and transmission electron microscopy images (TEM) were obtained with a Hitachi S-2600N (using a secondary electron scintillator as detector) and a JEOL 1200 EX microscope, respectively. For the purpose of TEM analysis, the sample particles were dispersed in ethanol and then dropped onto copper grids with porous carbon films. The specific surface area and pore structure parameters of the materials studied were determined from the measurements of nitrogen adsorption-desorption at $77 \mathrm{~K}$ with the use of a Micromeritics ASAP 2020 V3.00 H unit. Prior to the sorption experiment, the sample (about $40 \mathrm{mg}$ ) was evacuated under vacuum at $523 \mathrm{~K}$ for $12 \mathrm{~h}$. In view of applications of the present Cu-RMSN materials as catalysts, the number of acid sites was assessed and attributed to the number of accessible copper atoms at the solid surface by assuming that pure silica displayed a very limited number of acid sites. ${ }^{26}$ Measurements of the two-cycle adsorption of gaseous ammonia at $373 \mathrm{~K}$ were achieved with the aid of a Micromeritics ASAP 2010 V2.02 and the results served to determine the number of acid sites in the material surface. The sample was outgassed prior to the measurements at $473 \mathrm{~K}$ for $2 \mathrm{~h}$ at a rate of $5 \mathrm{~K} \cdot \mathrm{min}^{-1}$. Afterward, the two-cycle adsorption experiments were performed under equilibrium adsorption conditions at $373 \mathrm{~K}$ with gaseous $\mathrm{NH}_{3}$ of ultrahigh purity. At the end of the first adsorption cycle, the sample was outgassed under vacuum at $373 \mathrm{~K}$ for $60 \mathrm{~min}$ and a second adsorption cycle was then performed at the same temperature. It is usually assumed that the linear section distinguished in both isotherms at higher equilibrium pressures may be ascribed to physical adsorption on nonreactive surface sites. Therefore, the two straight lines were extrapolated to zero pressure. The difference in the amount adsorbed for points at which the curves reach zero pressure was ascribed to the irreversible chemisorption of basic $\mathrm{NH}_{3}$ at the solid-gas interface and thereby to the total number of acid sites at the solid surface per unit mass of the adsorbent. 
Small-angle powder X-ray diffraction (XRD) measurements were performed with a PANalytical X'Pert MPD (Philips 1710) diffractometer controlled by a computer. The XRD patterns were collected using a $\mathrm{Cu} \mathrm{K} \alpha$ radiation, the $2 \theta$ diffraction angles being recorded at a rate of $0.5^{\circ} \mathrm{min}^{-1}$ in the range from $1^{\circ}$ to $7^{\circ}$. For some selected samples containing copper, the XRD experiment was continued in the $2 \theta$ range up to $60^{\circ}$. Temperature-programmed reduction and metal dispersion experiments were also carried out in the temperature range from 35 to $800{ }^{\circ} \mathrm{C}$ (heating rate $2 \mathrm{~K} \mathrm{~min}^{-1}$ ) under a flow of $\mathrm{H}_{2}$ utilizing a Micromeritics AutoChem 2910 equipped with a Dewar flask containing cold medium to trap water. ${ }^{1} \mathrm{H}$ NMR spectra were obtained at $297 \mathrm{~K}$ using an AC 300L Bruker spectrometer operating at a Larmor frequency of $69.19 \mathrm{MHz}(15000$ signal acquisitions, recycle delay of $8 \mathrm{~s}$ between $\pi / 2$ pulses). NMR spectra were recorded at room temperature with the appropriate deuterated solvent $\left(\mathrm{CDCl}_{3}\right.$ or DMSO- $\left.d_{6}\right)$. Chemical shifts $(\delta)$ of ${ }^{1} \mathrm{H}$ NMR and ${ }^{13} \mathrm{C}$ NMR spectra are reported in ppm relative to residual solvent signals $\left(\mathrm{CHCl}_{3}\right.$ in $\mathrm{CDCl}_{3}: \delta=7.27 \mathrm{ppm}$ for ${ }^{1} \mathrm{H}$ and $\mathrm{CDCl}_{3}: \delta=77.04$ ppm for ${ }^{13} \mathrm{C}$ NMR); data are reported as br = broad, $\mathrm{s}=$ singlet, $\mathrm{d}=$ doublet, $\mathrm{t}=$ triplet, $\mathrm{q}=$ quartet, $\mathrm{m}=$ multiplet; $J$ values are given in $\mathrm{Hz}$. ${ }^{1} \mathrm{H}$ and ${ }^{13} \mathrm{C}$ NMR spectra were registered at 300 or $400 \mathrm{MHz}$. The nature of analytically pure final products was assessed by comparison of their ${ }^{1} \mathrm{H}$ NMR data previously described in the literature and by their fragmentation in the LC/MS equipment. Conversions and yields were calculated by adding $\mathrm{CH}_{2} \mathrm{Br}_{2}(0.25 \mathrm{mmol} ; 20 \mu \mathrm{L})$ as an internal standard. Analytical high performance liquid chromatography (HPLC) was performed using a CHROMOLITH RP18 column $(50 \times 4,6$ $\mathrm{mm}$ ), variable wavelength diode, flow of $5 \mathrm{~mL} \mathrm{~min}{ }^{-1}$, linear gradient $\mathrm{CH}_{3} \mathrm{CN}$ in water $0-100 \%(+0.1 \% \mathrm{TFA})$ in $4.5 \mathrm{~min}$. The LC-MS analysis was performed by means of HPLC Waters Alliance 2695 (UV Waters 2489), column Onyx C18 $(25 \times 4.6 \mathrm{~mm})$, flow of $3 \mathrm{~mL} \mathrm{~min}^{-1}$, linear gradient $\mathrm{CH}_{3} \mathrm{CN}$ in water $0-100 \%\left(+0.1 \% \mathrm{HCO}_{2} \mathrm{H}\right)$ in $2.5 \mathrm{~min}$. Mass spectra (electrospray ionization mode, ESI-MS) were recorded on a Micromass (Manchester, UK) Q-TOF quadrupole mass spectrometer fitted with an electrospray interface. The mass spectrometer was calibrated in the positive- and negative-ion ESI modes. Samples were dissolved in a $\mathrm{H}_{2} \mathrm{O} / \mathrm{CH}_{3} \mathrm{CN}(50 / 50 \mathrm{v} / \mathrm{v})$ mixture. Microwave-assisted reactions were performed in sealed vessels with a Biotage Initiator 60 EXP instrument. The temperature was measured with an IR sensor at the outer surface of the reaction vial. Thermal gravimetric analysis (TGA) and differential thermal analysis (DTA) were carried out on a SETARAM Labsys apparatus in order to study changes in the chemical properties of the pristine samples and those containing $\mathrm{Cu}$ species as a function of increasing temperature. A given mass of solid sample was placed in a $80 \mu \mathrm{L}$ alumina crucible. The TGA and DTA curves were obtained up to $873 \mathrm{~K}$ in flowing nitrogen using a constant heating rate of $10^{\circ} \mathrm{min}^{-1}$. Ultraviolet-visible (UVvis) absorption spectra were collected with an UV-visible Jasco V-670 spectrophotometer. Leaching of $\mathrm{Cu}$ from $\mathrm{Cu}$-RMSN was assessed by inductive coupled plasma (ICP-MS, Agilent 7700x) analysis of the aqueous phase; the instrument detection limit for copper was below 30 ppt.

Catalyst Preparation. The $\mathrm{Cu}$-doped mesoporous silica rodshaped nanoparticles (Cu-RMSN) were prepared by a one-step synthesis-functionalization procedure, based on the co-condensation of (3-aminopropyl)triethoxysilane (APTES) and tetraethyl orthosilicate (TEOS) as a silicon source. In a typical synthesis, $0.05 \mathrm{~g}$ of hexadecyltrimethylammonium bromide (CTAB) was dissolved in 50 $\mathrm{mL}$ of deionized water under continuous stirring at room temperature. Then, $\mathrm{Cu}(\mathrm{acac})_{2}$, previously dissolved in $200 \mu \mathrm{L}$ of chloroform, was added to the above-mentioned mixture under vigorous stirring at 343 $\mathrm{K}$. This step corresponded to the solubilization of the copper precursor in micellar aggregates. $575 \mu \mathrm{L}$ TEOS and $80 \mu \mathrm{L}$ APTES were subsequently added to the solution at $313 \mathrm{~K}$ under intensive stirring. Hydrolysis and polycondensation steps were carried out at $\mathrm{pH}$ adjusted to 12 with sodium hydroxide. The molar composition of the final solution was as follows: (i) CTAB: $\mathrm{NaOH}$ :TEOS:APTES:Cu$(\mathrm{acac})_{2}=1: 9.7 \times 10^{-4}: 1.9 \times 10^{-2}: 2.5 \times 10^{-4}$; (ii) $X\left(X=2.0 \times 10^{-5}\right.$; $\left.2.7 \times 10^{-5} ; 3.4 \times 10^{-5}\right)$. The colloidal mixture was stirred for 2 additional hours. Afterward, the resulting white precipitate was recovered by centrifugation and dried in air at $323 \mathrm{~K}$. The template was eliminated from Cu-RMSN by air calcinations at $723 \mathrm{~K}$ for $8 \mathrm{~h}$ with a heated rate of $1 \mathrm{~K} \mathrm{~min}^{-1}$. A fine slightly blue powder of $\mathrm{Cu}$ RMSN was finally obtained. The three samples were designated $\mathrm{Cu}-$ RMSN- $X$ with $X$ corresponding to the initial Si:Cu ratio (i.e., 190, 112, 84 ) in the gel synthesis. The pure siliceous material designated as RMSN was prepared using the previous procedure except that the $\mathrm{Cu}$ source was excluded from the gel synthesis.

General Procedure for the One-Pot Synthesis of 1,4Disubstituted-1,2,3-triazoles under Microwave Irradiation. Cu-RMSN catalyst (35 mg, $1.25 \mathrm{~mol} \%$ ) was charged in a microwave Pyrex vial and activated throughout gassing under vacuum at $473 \mathrm{~K}$ for $8 \mathrm{~h}$. Then, the appropriate alkyl or benzyl halide $(0.2 \mathrm{mmol})$, alkyne $(0.2 \mathrm{mmol})$, sodium azide $(0.2 \mathrm{mmol})$, and sodium ascorbate $(0.2$ mmol) were added, together with about $2 \mathrm{~mL}$ of water. The reaction mixture was heated under microwave irradiation ${ }^{51}$ with constant stirring $(900 \mathrm{rpm})$ at $333 \mathrm{~K}$ for the specified time. At the end of the reaction, $\mathrm{CH}_{2} \mathrm{Cl}_{2} / \mathrm{Et}_{2} \mathrm{O}(1: 4 \mathrm{v} / \mathrm{v}, 5 \mathrm{~mL} \times 3)$ was added into the vial and the heterogeneous system was again stirred for $1 \mathrm{~min}$. Then, the upper organic layers were recovered by decantation, dried on $\mathrm{MgSO}_{4}$, filtered, and evaporated under vacuum to collect the corresponding 1,4-disubstituted-1,2,3-triazole as a white solid.

General Procedure for the Cu-RMSN Catalyst Recycling. To get rid of all water-soluble species from the catalyst surface, the catalyst was washed three times with $3 \times 5 \mathrm{~mL}$ of water added under constant stirring to a given Pyrex vial containing the catalyst issued from the previous run. The aqueous phase was removed each time by centrifugation and at least the catalyst was lyophilized. The resulting solid catalyst was reactivated as described previously, before being reused in a subsequent run following the same operating procedure.

\section{RESULTS AND DISCUSSION}

As described previously, ${ }^{27}$ the one-pot synthesis-functionalization approach allowing in situ metal incorporation in mesoporous silica led to $\mathrm{Cu}$-containing nanosized silica objects having different morphologies. Figure 1 shows SEM micro-

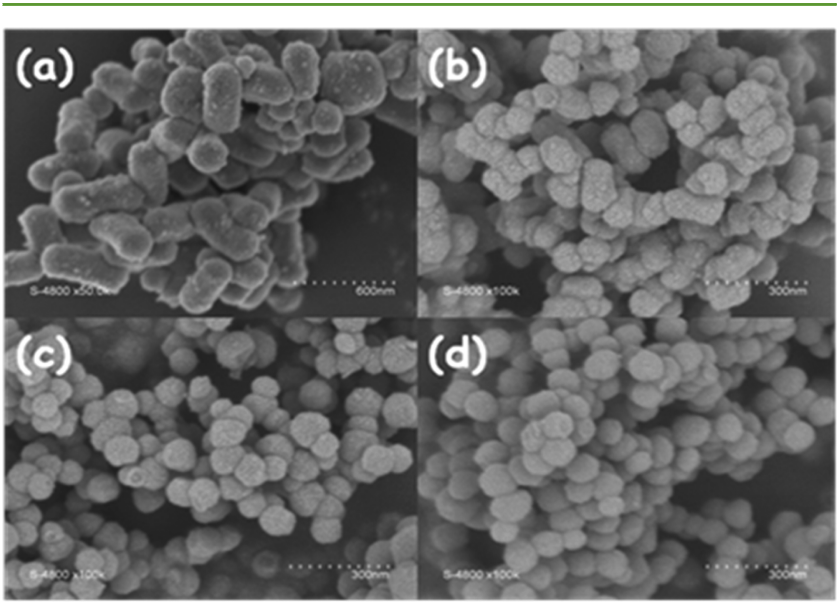

Figure 1. Scanning electron microscope images of nanosized silica objects prepared by a one-pot synthesis-functionalization method and containing different amounts of copper, as defined by the following molar Si:Cu ratio: (a) purely siliceous sample, (b) $\mathrm{Si}: \mathrm{Cu}=190,(\mathrm{c})$ $\mathrm{Si}: \mathrm{Cu}=112$, and $(\mathrm{d}) \mathrm{Si}: \mathrm{Cu}=84$.

graphs of the resulting particles, which are either rod-like or spherical, depending on the amount of metal incorporation dissolved in chloroform. Apparently, the rod-to-sphere shape transition was favored by higher copper contents. Irrespective of their shape, the particles appeared quite homogeneous in size. 

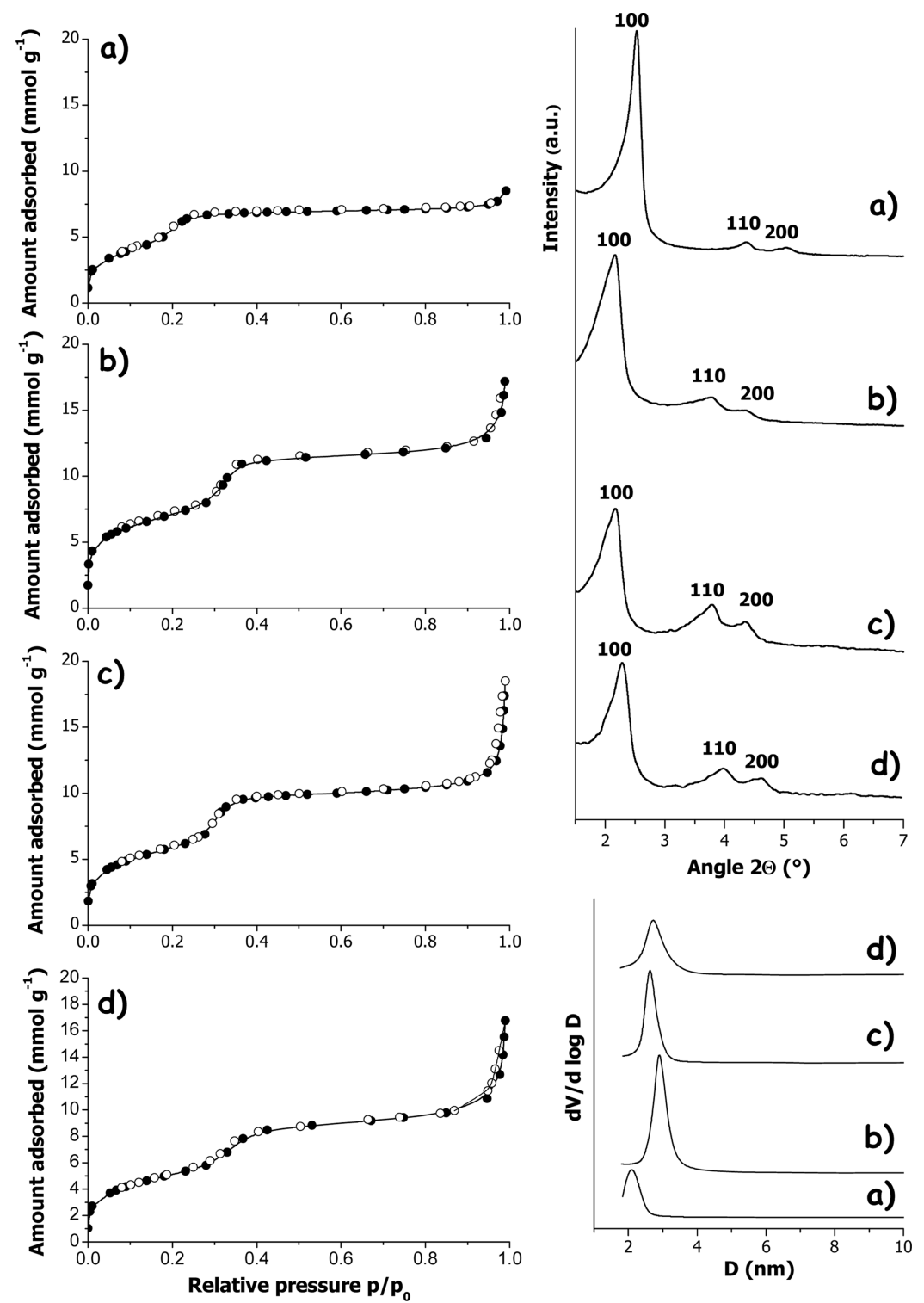

Figure 2. Adsorption-desorption isotherms of gaseous nitrogen at $77 \mathrm{~K}$ (4 left panels), powder XRD patterns (upper-right panel), and BJH pore size distributions based on the desorption branch of $\mathrm{N}_{2}$ adsorption isotherms (lower-right panel) for silica materials containing different amounts of copper, as defined by the following molar Si:Cu ratio: (a) purely siliceous sample, (b) Si:Cu = 190, (c) Si:Cu = 112, and (d) Si:Cu = 84.

The appropriate image processing gave the following size parameters: purely siliceous particles were $450-500 \mathrm{~nm}$ in length and 160-190 $\mathrm{nm}$ in width; rod-like particles with the smallest $\mathrm{Cu}$ content were $160-200 \mathrm{~nm}$ in length and $80-100$ $\mathrm{nm}$ in width; spherical particles were in the 75-110 and 75$100 \mathrm{~nm}$ diameter range with a $\mathrm{Si}: \mathrm{Cu}$ ratio of 112 and 84, respectively. This indicated that the increased copper incorporation resulted in the concomitant decrease in the particle size, the effect being less pronounced within the spherical shape category.

Changes in the pore arrangement and porosity parameters could be followed by the adsorption-desorption isotherms analysis of gaseous nitrogen at $77 \mathrm{~K}$, powder XRD patterns, and $\mathrm{BJH}$ pore size distributions as reported in Figure 2. The shape of the nitrogen adsorption curves was characteristic of silica materials possessing intra particle tubular mesopores at the lower end of the mesoporous regime, some residual inter particle porosity ( $\mathrm{Cu}$-containing spherical particles in particular), and a significant external surface area. ${ }^{2,52-54}$ A gradual increase in the amount of gaseous nitrogen adsorbed in the capillary condensation region indicated the intra particle mesopores were somehow heterogeneous, especially for the sample, which contained the highest copper amount. This heterogeneity was illustrated by the width of the $\mathrm{BJH}$ distribution curves. An additional characterization procedure based on the $\alpha_{\mathrm{S}}$-method was performed to determine the "average" pore structure (see Figure S1 in the Supporting Information), thus providing the following parameters: the micropore volume, $V_{\text {mic }}$ the area of the mesopore walls, $S_{\text {mes }}$, the external surface, $S_{\text {ext }}$, and the volume of mesopores, $V_{\text {mes }}$, as well as the mean pore diameter, $d_{\mathrm{m}}$, calculated assuming a cylindrical pore shape. ${ }^{53,55}$ The X-ray diffraction patterns clearly showed the first-order scattering peak $\left(d_{100}\right)$, whereas the low intensity (110) and (200) peaks were still distinguishable from the instrumental noise.

These XRD results corresponded to a 2D hexagonal mesostructure with a $p 6 \mathrm{~mm}$ space group. Therefore, the porosity of the four silica samples might be qualified of the 
MCM-41 type. All textural parameters inferred from the analysis of XRD patterns and $\mathrm{N}_{2}$ adsorption-desorption isotherms are reported in Table 1. It was observed that copper

Table 1. Surface Area and Pore Structure Parameters of the Calcined Silica Particles Containing Various Initial Amounts of Copper $^{a}$

\begin{tabular}{lllll}
\multicolumn{1}{c}{ Si:Cu ratio } & no $\mathrm{Cu}$ & 190 & 112 & 84 \\
$d_{100}$ spacing $(\mathrm{nm})$ & 3.5 & 4.10 & 4.01 & 3.87 \\
$S_{\mathrm{BET}}\left(\mathrm{m}^{2} \mathrm{~g}^{-1}\right)$ & 410 & 485 & 570 & 420 \\
$d_{\mathrm{BJH}}(\mathrm{nm})$ & 2.06 & 2.90 & 2.61 & 2.69 \\
$V_{\text {mic }}\left(\mathrm{cm}^{3} \mathrm{~g}^{-1}\right)$ & 0.02 & 0.03 & 0.05 & 0.02 \\
$S_{\text {ext }}\left(\mathrm{m}^{2} \mathrm{~g}^{-1}\right)$ & 15 & $47^{a}$ & $40^{a}$ & $54^{a}$ \\
$S_{\text {mes }}\left(\mathrm{m}^{2} \mathrm{~g}^{-1}\right)$ & 275 & $326^{a}$ & $395^{a}$ & $294^{a}$ \\
$V_{\text {mes }}\left(\mathrm{cm}^{3} \mathrm{~g}^{-1}\right)$ & 0.20 & $0.30^{a}$ & $0.32^{a}$ & $0.26^{a}$ \\
$d_{\mathrm{m}}(\mathrm{nm})$ & 3 & $3.6^{a}$ & $3.3^{a}$ & $3.6^{a}$
\end{tabular}

${ }^{a}$ Because the ascending part of the adsorption isotherm and the hysteresis loop at relative pressures close to unity do not allow these parameters to be determined unambiguously by applying the $\alpha_{\mathrm{S}}$-plot procedure, the values reported in the table should be treated with caution and only general trends are reliable. ${ }^{a}$ As indicated by the molar $\mathrm{Si}: \mathrm{Cu}$ ratio.

incorporation during the one-pot synthesis had a positive effect on promoting porosity formation. In any case, the pore size and the specific surface area increased, compared to the corresponding textural parameters characterizing the pristine sample containing no metal at all. These trends depended on both the overall metal content and the particle morphology. The greatest values of the surface area and pore volume parameters were obtained for the $\mathrm{Si}: \mathrm{Cu}=112$ sample. The $\mathrm{Cu}$ containing rod-like particles (i.e., $\mathrm{Si}: \mathrm{Cu}=190$ ) had the largest porosity, providing easier access to the internal porous space. Obviously, the selection of materials for applications in heterogeneous catalysis cannot be limited only to textural parameters. Such other characteristics like nature, distribution, and accessibility of the surface reactive sites are also of great significance. Further catalytic tests in aqueous medium revealed the superior performance of the $\mathrm{Si}: \mathrm{Cu}=190$ sample. Therefore, the surface properties of this material are described in more details in the following section.

Surface Properties of the Cu-containing Rod-like Particles. Further confirmation of the quasi-hexagonal order in the arrangement of tubular pores on the surface of silica particles could be obtained from the analysis of TEM micrographs (Figure 3). Different regions showing more or less marked alignment of pore channels with a uniform crosssectional diameter were visible (Figure 3). For numerous rods orientated horizontally, pore channels parallel to each other were extended along the entire length of the particle. A few particles had adopted a vertical orientation on the TEM holder and a quasi-hexagonal alignment of uniform channels could be seen from the top. In some cases, partial "finger-print" structures were also observed indicating some disorders in the channel alignment. Moreover, no metal containing clusters could be revealed (Figure 3), which points out a good dispersion of the copper species in the silica matrix.

More information about the nature and location of the copper species was obtained from the diffuse reflectance UVvis spectrum, powder $\mathrm{XRD}$ pattern in the wide-range region, $\mathrm{H}_{2}$-TPR profile, as well as TGA/TDA profiles acquired from the $\mathrm{Si}: \mathrm{Cu}=190$ sample (Figure 4). The UV-vis spectrum

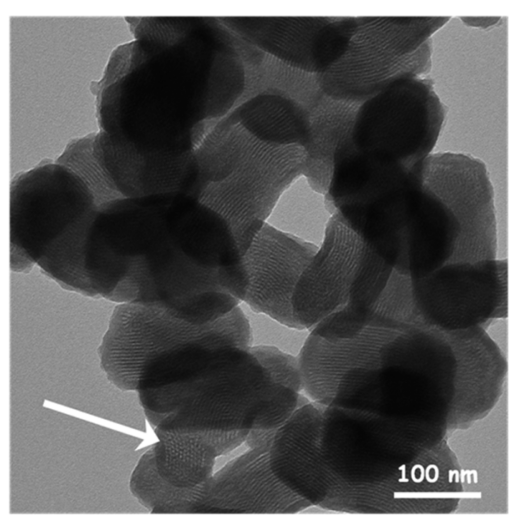

Figure 3. Transmission electron micrographs of nanosized rod-like silica objects containing copper $(\mathrm{Si}: \mathrm{Cu}=190)$. The white arrow indicates a region where a particle is oriented vertically thus showing a quasi-hexagonal alignment of pore channels.

displayed features similar to those observed previously: ${ }^{27}$ a main band representing a right-skewed peak with a maximum at about $225 \mathrm{~nm}$, a weak and very broad band at high wavelength values beyond $600 \mathrm{~nm}$ (Figure 4a). The main band could be considered as indicative of the ligand-to-metal charge transfer between $\mathrm{Cu}(\mathrm{II})$ ion and oxygen in mononuclear species located at the internal surface. ${ }^{27,56}$ The XRD pattern showed no peak in the $2 \theta$ range between $30^{\circ}$ and $60^{\circ}$ (Figure $4 \mathrm{~b}$ ), which argues against the presence of extra-framework metal oxide crystalline aggregates within the material. ${ }^{57}$ The TGA profile of the calcined $\mathrm{Cu}-\mathrm{RMSN}$ displayed no weight loss that could be ascribed to the decomposition of remaining surfactant (Figure $4 \mathrm{~d}$ ). The weight loss that occurred at temperatures close to 373 $\mathrm{K}$ could be assigned to the release of physisorbed water. The TPR profile showed a broad and asymmetric peak with a temperature at maximum hydrogen consumption $T_{\mathrm{m}}$ around $418 \mathrm{~K}$ (Figure 4c), usually attributed to the reduction of $\mathrm{Cu}$ (II) or finely dispersed $\mathrm{CuO}$ to copper metal in the channels of MCM-41 materials. ${ }^{19,20}$ Because the temperature of reduction was lower than that previously recorded for the spherical silica particles achieved by the same synthesis-functionalization procedure (i.e., $485 \mathrm{~K}^{27}$ ), it appeared that, in the present case, copper oxide was located in readily accessible positions well dispersed at the pore surface. Moreover, on the basis of the results of EDX and those of two-cycle ammonia adsorption onto Cu-RMSN ( $\mathrm{Si}: \mathrm{Cu}=190)$ (Figure 5), the accessibility of copper surface sites was evaluated by assuming that the ratio between the number of $\mathrm{Cu}$ atoms calculated from the number of acid sites, $N_{\text {acid }}$ and the theoretical number of $\mathrm{Cu}$ atoms calculated from the EDX results corresponded to the number of $\mathrm{Cu}$ atoms accessible to gaseous $\mathrm{NH}_{3}$ molecules. In the case of $\operatorname{RMSN}(\mathrm{Si}: \mathrm{Cu}=190)$, the $N_{\text {acid }}$ value was of $70 \mu \mathrm{mol} \mathrm{g}^{-1}$ (i.e., $4.4 \mathrm{mg}$ of $\mathrm{Cu}$ per gram of catalyst). When ascribed to the number of accessible copper atoms, this gave $85 \%$ accessibility with respect to the total number of copper atoms.

Catalytic Performance of the Cu-containing Rod-like Particles. The rod-like mesoporous silica nanoparticles with copper functionalities at the pore surface (Cu-RMSN), achieved in a one-step approach, were evaluated as nanocatalyst in the eco-friendly version of the Huisgen 1,3-dipolar cycloaddition (click reaction) leading to 1,4-disubstituted-1 $\mathrm{H}$ 1,2,3-triazoles. The regioselectivity was studied by making use of organic halides and terminal alkynes with sodium azide in water under microwave irradiation. This multicomponent 

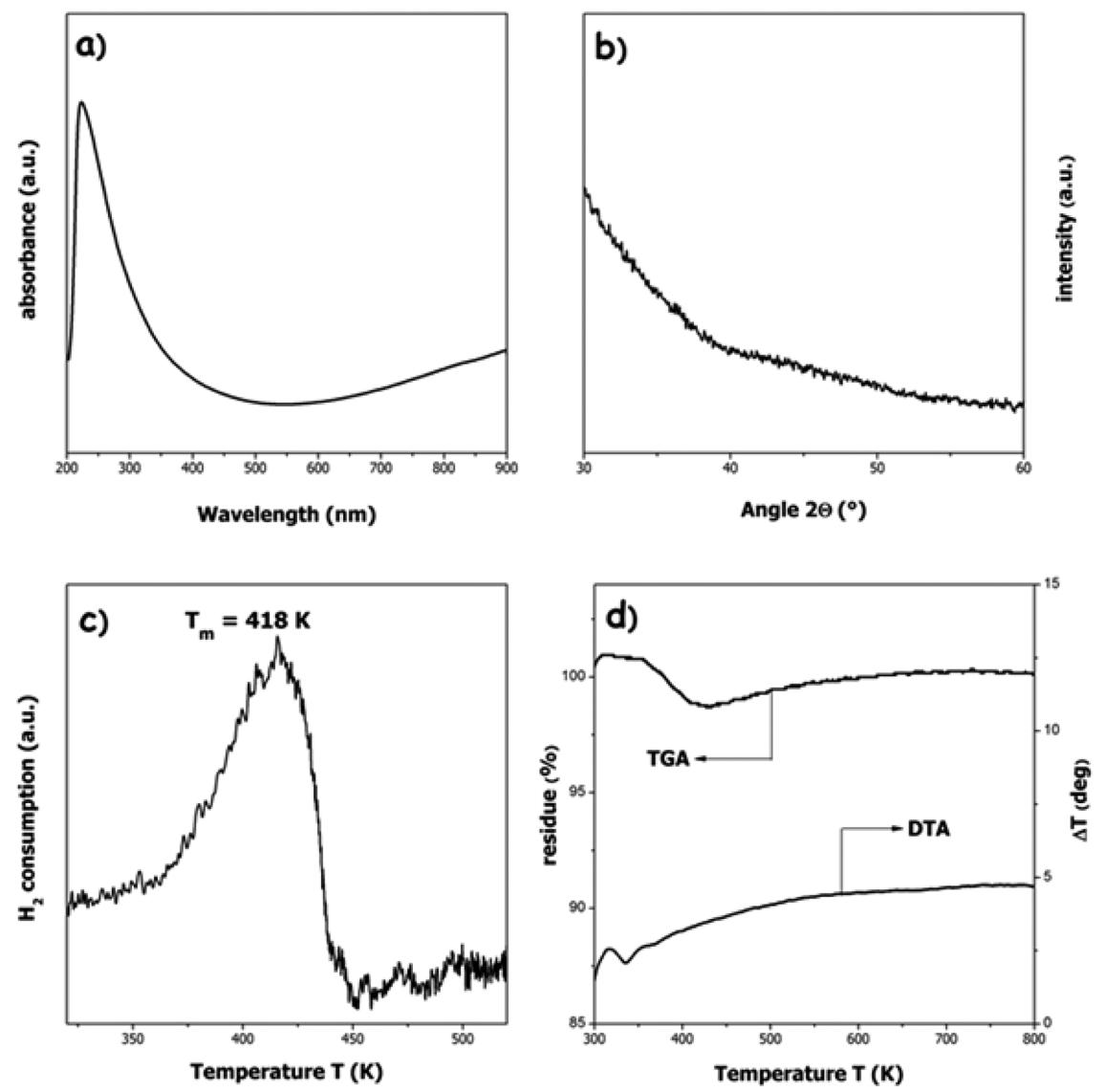

Figure 4. (a) Diffuse reflectance UV-vis spectrum; (b) wide-angle range X-ray powder diffraction pattern; (c) temperature-programmed desorption $\left(\mathrm{H}_{2}\right.$-TPR) profile; (d) results of thermogravimetric analysis for the rod-like silica particles containing copper $(\mathrm{Si}: \mathrm{Cu}=190)$.

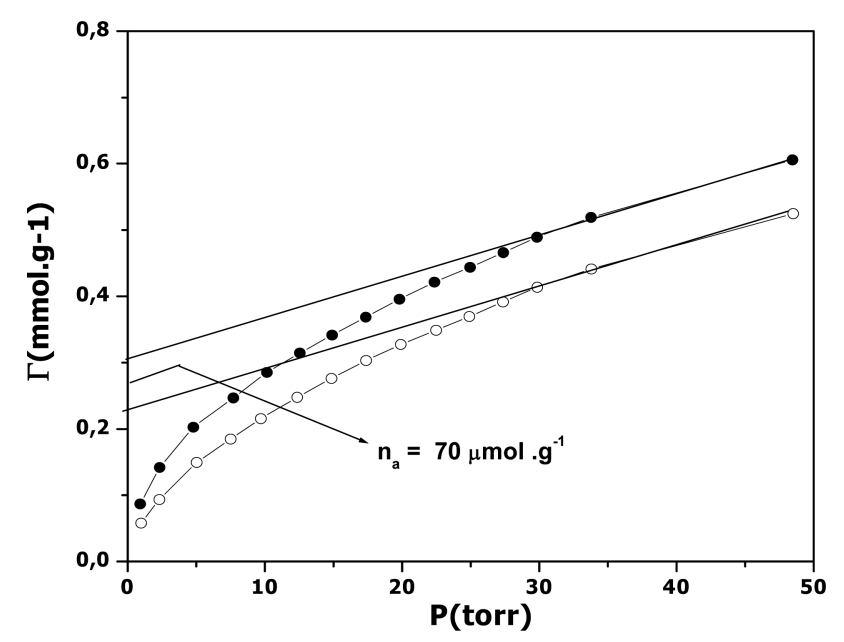

Figure 5. First-cycle $(\bullet)$ and second-cycle $(O)$ adsorption isotherms for gaseous ammonia onto R-CuMSN $(\mathrm{Si}: \mathrm{Cu}=190)$ at $373 \mathrm{~K}$.

reaction followed a two reactions cascade where the Cu-RMSN promoted first the nucleophilic substitution for the in situ formation of the organic azide and then catalyzed cycloaddition. $^{44,58}$ This avoided the isolation of organic azides (generally stable either in water or in the presence of oxygen under most reaction conditions), some lower alkyl azides decomposing rapidly with a risk of explosion. ${ }^{59}$ As representative substrates, benzyl bromide and phenyl acetylene were added in stoichiometric amounts to optimize the reaction conditions with $\mathrm{NaN}_{3}$ under microwave irradiation in aqueous medium (Table 2). Because the reaction had to be performed in the absence of catalyst, no triazole product was observed (Table 2, entry 1). As expected, ${ }^{44}$ the solvolysis product benzyl alcohol was formed. By irradiating the mixture for $30 \mathrm{~min}$ at 60 ${ }^{\circ} \mathrm{C}$, in the presence of $\mathrm{Cu}-\mathrm{RMSN}$ catalyst $(1.25 \mathrm{~mol} \%)$, the corresponding 1,4-disubstituted-1H-1,2,3-triazole was regioselectively obtained with a high yield (97\%, entry 2 ). The $\mathrm{Cu}-$ RMSN catalyst played a double role: it catalyzed the reaction $^{45,60}$ and avoided the formation of 1,5-disubstituted triazolyl regioisomer that had never been observed in the crude mixture, ${ }^{29,61}$ as previously reported. However, shortening the reaction time to $15 \mathrm{~min}$ decreased the yield (entry 3 ). In the absence of sodium ascorbate [ $\mathrm{Cu}(\mathrm{II})$ reducing agent], the reaction did not proceed as previously reported. ${ }^{29}$ This result ruled out the presence of $\mathrm{Cu}(\mathrm{I})$ entities dispersed in the silica matrix $^{34}$ during the oxidative homocoupling of alkynes leading to the diynes formation throughout the Glaser reaction, ${ }^{62}$ which had never been observed in the present study.

For comparison, the reaction was also carried out with the conventional catalyst $\mathrm{CuBr}$ salt at 7 and $49 \mathrm{~mol} \%$ of $\mathrm{Cu}$ content (entries 4 and 5) under the same reaction conditions, leading respectively to $29 \%$ and $96 \%$ yield of triazole 1 . This different behavior at low $\mathrm{Cu}$ concentrations compared to the conventional Huisgen copper-catalyzed reaction (CuAAC) in DMF solvent ${ }^{63,64}$ under microwave irradiation came from the biphasic character of the reactive medium. Indeed, copper ions were water-soluble, whereas the organic substrates were too hydrophobic to be soluble in aqueous solutions. As a result, the contact between the organic substrates and the copper species was limited and consequently the product yield was quite low. 
Table 2. Cu-RMSN-Catalyzed One-Pot Synthesis of 1,4-Disubstituted-1H-Triazoles

\begin{tabular}{|c|c|c|c|c|c|c|c|c|}
\hline \multirow[b]{3}{*}{ entry $^{a}$} & \multirow[b]{3}{*}{$\mathrm{R}^{1} / \mathrm{R}^{2}$} & \multirow[b]{3}{*}{ product } & \multirow[b]{3}{*}{$T\left({ }^{\circ} \mathrm{C}\right)$} & \multirow[b]{3}{*}{$t(\mathrm{~h})$} & $\begin{array}{l}\stackrel{\mathrm{NaN}_{3},[\mathrm{Cu}]}{\text { Sodium ascorbate }} \\
\underset{\mathrm{H}}{\mathrm{H} \mathrm{O}, 1 \mathrm{~h}-2 \mathrm{~h}} \\
\mathrm{MW}, 45-80^{\circ} \mathrm{C}\end{array}$ & 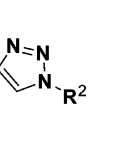 & & \\
\hline & & & & & \multicolumn{4}{|c|}{$[\mathrm{Cu}]$ catalyst } \\
\hline & & & & & {$[\mathrm{CuBr}] \mathrm{mol} \%$} & yield $(\%)^{b}$ & {$[\mathrm{Cu}-\mathrm{RMSN}] \mathrm{mol} \%$} & yield $(\%)^{b}$ \\
\hline 1 & $\mathrm{Ph} / \mathrm{PhCH}_{2}$ & 1 & 60 & 1 & 0 & 0 & 0 & 0 \\
\hline 2 & $\mathrm{Ph} / \mathrm{PhCH}_{2}$ & 1 & 60 & 0.5 & n.p. ${ }^{c}$ & n.p. ${ }^{c}$ & 1.25 & 97 \\
\hline 3 & $\mathrm{Ph} / \mathrm{PhCH}_{2}$ & 1 & 60 & 0.25 & n.p. ${ }^{c}$ & n.p. ${ }^{c}$ & 1.25 & 55 \\
\hline 4 & $\mathrm{Ph} / \mathrm{PhCH}_{2}$ & 1 & 60 & 1 & 7.0 & 29 & n.p. ${ }^{c}$ & n.p..$^{c}$ \\
\hline 5 & $\mathrm{Ph} / \mathrm{PhCH}_{2}$ & 1 & 60 & 1 & 49 & 96 & 1.25 & 98 \\
\hline 6 & $\mathrm{Ph} / \mathrm{CH}_{2} \mathrm{CO}_{2} \mathrm{Me}$ & 2 & 60 & 1 & 49 & 86 & 1.25 & 96 \\
\hline 7 & $n-\mathrm{Bu} / \mathrm{PhCH}_{2}{ }^{d}$ & 3 & 60 & 1 & 49 & 78 & 1.25 & 79 \\
\hline 8 & $\mathrm{Ph} / \mathrm{CH}_{2} \mathrm{CO}_{2} \mathrm{Bn}$ & 4 & 70 & 2 & 49 & 67 & 1.25 & 86 \\
\hline 9 & $\mathrm{Ph} / 2-\mathrm{I}-\mathrm{C}_{6} \mathrm{H}_{4} \mathrm{CH}_{2}$ & 5 & 45 & 1 & 49 & 63 & 1.25 & 86 \\
\hline 10 & $\mathrm{Ph} / n-\mathrm{Bu}^{e}$ & 6 & 80 & 2 & 49 & 61 & 1.25 & 69 \\
\hline 11 & $\mathrm{Ph} / \mathrm{sec}-\mathrm{Bu}^{e}$ & 7 & 80 & 2 & 49 & 69 & 1.25 & 73 \\
\hline
\end{tabular}

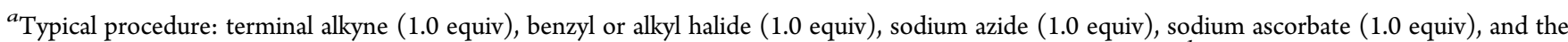
catalyst were suspended in water $(2 \mathrm{~mL})$ and heated under microwave for the indicated time and temperature. ${ }^{b}$ Yields were calculated by ${ }^{1} \mathrm{H}$ NMR using $\mathrm{CH}_{2} \mathrm{Br}_{2}(20 \mu \mathrm{L}, 0.25 \mathrm{mmol})$ as internal standard. ${ }^{c}$ n.p. $=$ not performed. ${ }^{d} 4.0$ equiv of benzyl bromide was used. ${ }^{e} 2.0$ equiv of alkyl bromide was used.

When increasing the amount of $\mathrm{CuBr}$ to $49 \%$ ( $\mathrm{Cu}$ content), the solubility limit was reached with the opportunity of substrate adsorption on the active $\mathrm{CuBr}$ particles dispersed in the aqueous solution. As a consequence, in order to compare the action of the Cu-RMSN catalyst with that of the conventional $\mathrm{CuBr}$ at $49 \mathrm{~mol} \%$, the selected reaction conditions were fixed at $1 \mathrm{~h}$ and $60{ }^{\circ} \mathrm{C}$ in aqueous solution, under microwave irradiation (entry 5).

The possibility of reacting phenyl acetylene with variation in the halide substrates compatible with the standard reaction conditions was subsequently explored. Aliphatic hex-1-yne reacted similarly to phenyl acetylene, yielding the corresponding triazoles with mostly the same yields and regioselectively (entry 7). The "click reaction" with 2-iodo-benzyl bromide was also efficient and not affected by the steric and electronic effects regarding the substitution on the ortho-position (entry 9) or by using functionalized alkyl bromides (entries 6 and 8). Primary or secondary alkyl bromides (entries 10 vs 11 ) reacted similarly, although the yields were slightly decreased. In all our study, $\mathrm{Cu}$ RMSN showed a higher efficiency and performance than $\mathrm{CuBr}$. Indeed, in all cases the yield of reactions performed with $\mathrm{Cu}$ RMSN was higher compared to that using $\mathrm{CuBr}$. This may be explained by the higher copper dispersion at the pore surface, accounting for the higher accessibility of the active sites, as inferred from the chemisorption measurements (Figure 5). Indeed, $\mathrm{CuBr}$ was dissolved in the aqueous phase and was not in direct contact with the nonaqueous miscible substrates. In the case of $\mathrm{Cu}-\mathrm{RMSN}$, the substrates were adsorbed at the pore surface of the mesoporous nanoparticles in the vicinity of the $\mathrm{Cu}$ catalytic active sites.

Reusability of the Cu-RMSN Catalyst. Considering the great economic and environmental added values of heterogeneous catalysts, it is important to examine their ease of separation, recoverability and reusability. In order to evaluate the reuse of the mesoporous $\mathrm{Cu}-\mathrm{RMSN}$ catalyst we investigated the "click synthesis" of 1-benzyl-4-phenyl-1H-1,2,3-triazole 1 (Table 2, entry 5) and we adjusted the experimental conditions of the present system. After each cycle, the reaction was followed by extraction of the products and unreacted substrates, and the catalyst was washed with water, lyophilized, and then reactivated before performing the next run, without any further purification. The performance of the recycled catalyst was tested up to ten successive runs without significant yield loss of the reaction products (Figure 6). These results pointed out the

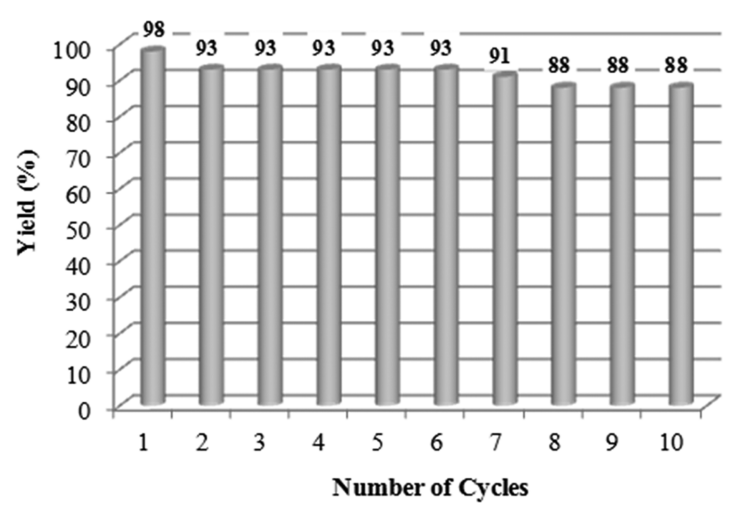

Figure 6. Recyclability of the Cu-RMSN catalytic system during repeated synthesis.

catalytic efficiency of the recovered mesoporous Cu-RMSN, retaining the same activity throughout all reaction cycles, thereby indicating the high stability of the copper species at the pore surface. Moreover, at the end of the first cycle, the reaction mixture contained only $523 \mathrm{ppb}$ of $\mathrm{Cu}$, as indicated by the ICPMS analysis. These copper species in solution were released during the reaction and originated from leaching. This amount corresponded to $0.16 \%$ molar of the copper initially present in $\mathrm{Cu}-\mathrm{RMSN}$ and did not disturb catalysis of the reaction, since $7 \%$ of the $\mathrm{Cu}$ content was globally ineffective (Table 2, entry 4). The copper amount after the second cycle was of $61 \mathrm{ppb}$ and only of $9 \mathrm{ppb}$ after 10 successive cycles, indicating the high stability of the Cu-RMSN catalyst (Figure 6). It seems that the copper species localized at the mesopore surface in the rod-like MSN help to prevent the metal leaching and to preserve the high efficiency of the catalyst recycling, while keeping the regioselectivity of the reactions. 


\section{CONCLUSIONS}

The insertion of $\mathrm{Cu}$ into the mesopores of rod-shaped mesoporous silica nanoparticles has been achieved with good control of morphological and textural properties. The TPR analysis of $\mathrm{Cu}$-RMSN showed that the well-dispersed $\mathrm{Cu}$ species were confined inside the mesopores. The Cu-RMSN sample with a low catalyst loading $(1.25 \%)$ was used to prepare regioselectively 1,4-disubstituted-1H-1,2,3-triazoles with a high yield via an eco-friendly multicomponent approach, in aqueous media and using microwave heating. The intrinsic and optimal atom economy for the CuAAC reaction, the use of water as a sustainable solvent, the lower catalytic charge compared to the previous reports with similar systems, the solvent economy related to the recovery of the final pure product, together with the ability to reuse the catalyst for at least ten subsequent runs without a marked loss of activity, contributed to the "green" character of the present catalytic process. In the future, preparation of enlarged mesopores represents a challenge to be considered to generalize the catalytic applications of such rod-like mesoporous silica nanoparticles to other reagents and substrates.

\section{AUTHOR INFORMATION}

\section{Corresponding Authors}

*C. Charnay. E-mail: clarence.charnay@umontpellier.fr.

*E. Colacino. E-mail: evelina.colacino@umontpellier.fr.

\section{Notes}

The authors declare no competing financial interest.

\section{REFERENCES}

(1) Tsai, C.-H.; Chen, H.-T.; Althaus, S. M.; Mao, K.; Kobayashi, T.; Pruski, M.; Lin, V. S.-Y. Rational Catalyst Design: A Multifunctional Mesoporous Silica Catalyst for Shifting the Reaction Equilibrium by Removal of Byproduct. ACS Catal. 2011, 1, 729-732.

(2) Carrillo, A. I.; Serrano, E.; Luque, R.; García-Martínez, J. Microwave-assisted catalysis by iron oxide nanoparticles on MCM-41: Effect of the support morphology. Appl. Catal., A 2013, 453, 383-390.

(3) Liu, D.-J.; Wang, J.; Ackerman, D. M.; Slowing, I. I.; Pruski, M.; Chen, H.-T.; Lin, V. S.-Y.; Evans, J. W. Interplay between Anomalous Transport and Catalytic Reaction Kinetics in Single-File Nanoporous Systems. ACS Catal. 2011, 1, 751-763.

(4) Chen, Y.; Zhang, F.; Fang, Y.; Zhu, X.; Zhen, W.; Wang, R.; Ma, J. Phosphotungstic acid containing ionic liquid immobilized on magnetic mesoporous silica rod catalyst for the oxidation of dibenzothiophene with $\mathrm{H}_{2} \mathrm{O}_{2}$. Catal. Commun. 2013, 38, 54-58.

(5) Yin, Y.; Liu, K.; Gao, M.; Zhang, L.; Su, H.; Zeng, S. Influence of the structure and morphology of $\mathrm{CuO}$ supports on the amount and properties of copper-cerium interfacial sites in inverse $\mathrm{CeO}_{2} / \mathrm{CuO}$ catalysts. J. Mol. Catal. A: Chem. 2015, 404-405, 193-203.

(6) Gao, D.; Duan, A.; Zhang, X.; Zhao, Z.; E, H.; Li, J.; Wang, H. Synthesis of NiMo catalysts supported on mesoporous Al-SBA-15 with different morphologies and their catalytic performance of DBT HDS. Appl. Catal., B 2015, 165, 269-284.

(7) Carrillo, A. I.; Serrano, E.; Luque, R.; García Matínez, J. Introducing catalytic activity in helical nanostructures: microwave assisted oxathioacetalisation catalysed by $\mathrm{Al}$-containing helical mesoporous silicas. Chem. Commun. 2010, 46, 5163-5165.

(8) Carrillo, A. I.; Serrano, E.; Serrano-Ruiz, J. C.; Luque, R.; GarciaMartinez, J. Helical Al- and Ce-MCM-41 materials as novel catalyst for acid and redox processes. Appl. Catal., A 2012, 435-436, 1-9.

(9) Minakuchi, H.; Nakanishi, K.; Soga, N.; Ishizuka, N.; Tanaka, N. Octadecylsilylated Porous Silica Rods as Separation Media for Reversed-Phase Liquid Chromatography. Anal. Chem. 1996, 68 (19), 3498-3501.

(10) Rambaud, F.; Vallé, K.; Thibaud, S.; Julián-López, B.; Sanchez, C. One-Pot Synthesis of Functional Helicoidal Hybrid OrganicInorganic Nanofibers with Periodically Organized Mesoporosity. Adv. Funct. Mater. 2009, 19 (18), 2896-2905.

(11) Trewyn, B. G.; Giri, S.; Slowing, I. I.; Lin, V. S. Y. Mesoporous silica nanoparticle based controlled release, drug delivery, and biosensor systems. Chem. Commun. 2007, No. 31, 3236-3245.

(12) Huang, X.; Teng, X.; Chen, D.; Tang, F.; He, J. The effect of the shape of mesoporous silica nanoparticles on cellular uptake and cell function. Biomaterials 2010, 31 (3), 438-448.

(13) Huang, X.; Li, L.; Liu, T.; Hao, N.; Liu, H.; Chen, D.; Tang, F. The Shape Effect of Mesoporous Silica Nanoparticles on Biodistribution, Clearance, and Biocompatibility in Vivo. ACS Nano 2011, 5 (7), 5390-5399.

(14) Xu, C.; Niu, Y.; Popat, A.; Jambhrunkar, S.; Karmakar, S.; Yu, C. Rod-like mesoporous silica nanoparticles with rough surfaces for enhanced cellular delivery. J. Mater. Chem. B 2014, 2 (3), 253-256.

(15) Yang, P.; Gai, S.; Lin, J. Functionalized mesoporous silica materials for controlled drug delivery. Chem. Soc. Rev. 2012, 41 (9), 3679-3698.

(16) Meng, H.; Yang, S.; Li, Z.; Xia, T.; Chen, J.; Ji, Z.; Zhang, H.; Wang, X.; Lin, S.; Huang, C.; Zhou, Z. H.; Zink, J. I.; Nel, A. E. Aspect Ratio Determines the Quantity of Mesoporous Silica Nanoparticle Uptake by a Small GTPase-Dependent Macropinocytosis Mechanism. ACS Nano 2011, 5 (6), 4434-4447.

(17) Fernandes, C. I.; Saraiva, M. S.; Nunes, T. G.; Vaz, P. D.; Nunes, C. D. Highly enantioselective olefin epoxidation controlled by helical confined environments. J. Catal. 2014, 309, 21-32.

(18) Bérubé, F.; Khadraoui, A.; Florek, J.; Kaliaguine, S.; Kleitz, F. A generalized method toward high dispersion of transition metals in large pore mesoporous metal oxide/silica hybrids. J. Colloid Interface Sci. 2015, 449, 102-114.

(19) Kargol, M.; Zajac, J.; Jones, D. J.; Rozière, J.; Steriotis, T.; Jiménez-López, A.; Rodríguez-Castellón, E. Copper- and SilverContaining Monolithic Silica-Supported Preparations for Selective Propene-Propane Adsorption from the Gas Phase. Chem. Mater. 2005, 17 (24), 6117-6127.

(20) Velu, S.; Wang, L.; Okazaki, M.; Suzuki, K.; Tomura, S. Characterization of MCM-41 mesoporous molecular sieves containing copper and zinc and their catalytic performance in the selective oxidation of alcohols to aldehydes. Microporous Mesoporous Mater. 2002, 54 (1-2), 113-126.

(21) Rascon, F.; Wischert, R.; Coperet, C. Molecular nature of support effects in single-site heterogeneous catalysts: silicavs.alumina. Chem. Sci. 2011, 2 (8), 1449-1456.

(22) Perego, C.; Millini, R. Porous materials in catalysis: challenges for mesoporous materials. Chem. Soc. Rev. 2013, 42 (9), 3956-3976.

(23) Taguchi, A.; Schüth, F. Ordered mesoporous materials in catalysis. Microporous Mesoporous Mater. 2005, 77 (1), 1-45.

(24) Valtchev, V.; Tosheva, L. Porous Nanosized Particles: Preparation, Properties, and Applications. Chem. Rev. 2013, 113 (8), 6734-6760.

(25) Huh, S.; Wiench, J. W.; Yoo, J.-C.; Pruski, M.; Lin, V. S. Y. Organic Functionalization and Morphology Control of Mesoporous Silicas via a Co-Condensation Synthesis Method. Chem. Mater. 2003, 15 (22), 4247-4256.

(26) Nyalosaso, J. L.; Derrien, G.; Charnay, C.; de Menorval, L.-C.; Zajac, J. Aluminium-derivatized silica monodisperse nanospheres by a one-step synthesis-functionalization method and application as acid catalysts in liquid phase. J. Mater. Chem. 2012, 22 (4), 1459-1468. 
(27) Derrien, G.; Charnay, C.; Zajac, J.; Jones, D. J.; Roziere, J. Copper-containing monodisperse mesoporous silica nanospheres by a smart one-step approach. Chem. Commun. 2008, 27, 3118-20.

(28) Huisgen, R. Kinetics and reaction mechanisms: Selected examples from the experience of forty years. Pure Appl. Chem. 1989, 61 (4), 613-628.

(29) Rostovtsev, V. V.; Green, L. G.; Fokin, V. V.; Sharpless, K. B. A Stepwise Huisgen Cycloaddition Process: Copper(I)-Catalyzed Regioselective "Ligation" of Azides and Terminal Alkynes. Angew. Chem., Int. Ed. 2002, 41 (14), 2596-2599.

(30) Tornøe, C. W.; Christensen, C.; Meldal, M. Peptidotriazoles on solid phase:[1, 2, 3]-triazoles by regiospecific copper (I)-catalyzed 1, 3dipolar cycloadditions of terminal alkynes to azides. J. Org. Chem. 2002, 67 (9), 3057-3064.

(31) Padwa, A. 1,3-Dipolar cycloaddition chemistry; Wiley-Interscience: New York, 1984; Vol. 1.

(32) Bock, V. D.; Hiemstra, H.; van Maarseveen, J. H. CuI-Catalyzed Alkyne-Azide "Click" Cycloadditions from a Mechanistic and Synthetic Perspective. Eur. J. Org. Chem. 2006, 2006 (1), 51-68.

(33) Mohammed, S.; Padala, A. K.; Dar, B. a.; Singh, B.; Sreedhar, B.; Vishwakarma, R. a.; Bharate, S. B. Recyclable clay supported Cu (II) catalyzed tandem one-pot synthesis of 1-aryl-1,2,3-triazoles. Tetrahedron 2012, 68, 8156-8162.

(34) Wang, Y.; Liu, J.; Xia, C. Insights into Supported Copper(II)Catalyzed Azide-Alkyne Cycloaddition in Water. Adv. Synth. Catal. 2011, 353 (9), 1534-1542.

(35) Lal, S.; Diez-Gonzalez, S. [CuBr(PPh3)3] for Azide-Alkyne Cycloaddition Reactions under Strict Click Conditions. J. Org. Chem. 2011, 76 (7), 2367-2373.

(36) Soltani Rad, M. N.; Behrouz, S.; Doroodmand, M. M.; Movahediyan, A. Copper-doped silica cuprous sulfate (CDSCS) as a highly efficient and new heterogeneous nano catalyst for $[3+2]$ Huisgen cycloaddition. Tetrahedron 2012, 68 (38), 7812-7821.

(37) Yamada, Y. M. A.; Sarkar, S. M.; Uozumi, Y. Amphiphilic SelfAssembled Polymeric Copper Catalyst to Parts per Million Levels: Click Chemistry. J. Am. Chem. Soc. 2012, 134 (22), 9285-9290.

(38) Buckle, D. R.; Outred, D. J.; Rockell, C. J. M.; Smith, H.; Spicer, B. A. Studies on v-triazoles. 7. Antiallergic 9-oxo-1H,9H-benzopyrano[2,3-d]-v-triazoles. J. Med. Chem. 1983, 26 (2), 251-254.

(39) Romero, A.; Liang, C.-H.; Chiu, Y.-H.; Yao, S.; Duffield, J.; Sucheck, S. J.; Marby, K.; Rabuka, D.; Leung, P. Y.; Shue, Y.-K.; Ichikawa, Y.; Hwang, C.-K. An efficient entry to new sugar modified ketolide antibiotics. Tetrahedron Lett. 2005, 46 (9), 1483-1487.

(40) Genin, M. J.; Allwine, D. A.; Anderson, D. J.; Barbachyn, M. R.; Emmert, D. E.; Garmon, S. A.; Graber, D. R.; Grega, K. C.; Hester, J. B.; Hutchinson, D. K.; Morris, J.; Reischer, R. J.; Ford, C. W.; Zurenko, G. E.; Hamel, J. C.; Schaadt, R. D.; Stapert, D.; Yagi, B. H. Substituent Effects on the Antibacterial Activity of Nitrogen-Carbon-Linked (Azolylphenyl)oxazolidinones with Expanded Activity Against the Fastidious Gram-Negative Organisms Haemophilus influenzae and Moraxella catarrhalis. J. Med. Chem. 2000, 43 (5), 953-970.

(41) Holla, B. S.; Mahalinga, M.; Karthikeyan, M. S.; Poojary, B.; Akberali, P. M.; Kumari, N. S. Synthesis, characterization and antimicrobial activity of some substituted 1,2,3-triazoles. Eur. J. Med. Chem. 2005, 40 (11), 1173-1178.

(42) Zhou, L.; Amer, A.; Korn, M.; Burda, R.; Balzarini, J.; De Clercq, E.; Kern, E. R.; Torrence, P. F. Synthesis and Antiviral Activities of 1,2,3-triazole Functionalized Thymidines: 1,3-dipolar Cycloaddition for Efficient Regioselective Diversity Generation. Antiviral Chem. Chemother. 2005, 16 (6), 375-383.

(43) Alvarez, R.; Velazquez, S.; San-Felix, A.; Aquaro, S.; Clercq, E. D.; Perno, C.-F.; Karlsson, A.; Balzarini, J.; Camarasa, M. J. 1,2,3Triazole-[2,5-Bis-O-(tert-butyldimethylsilyl)-.beta.-D-ribofuranosyl]$3^{\prime}$-spiro-5" $5^{\prime \prime}\left(4^{\prime \prime}\right.$-amino- $1^{\prime \prime}, 2^{\prime \prime}$-oxathiole $2^{\prime \prime}, 2^{\prime \prime}$-dioxide) (TSAO) Analogs: Synthesis and Anti-HIV-1 Activity. J. Med. Chem. 1994, 37 (24), 4185-4194.

(44) Bénéteau, V.; Olmos, A.; Boningari, T.; Sommer, J.; Pale, P. Zeo-click synthesis: CuI-zeolite-catalyzed one-pot two-step synthesis of triazoles from halides and related compounds. Tetrahedron Lett. 2010, 51 (28), 3673-3677.

(45) Radatz, C. S.; Soares, L. D. A.; Vieira, E. R; Alves, D.; Russowsky, D.; Schneider, P. H. Recoverable $\mathrm{Cu} / \mathrm{SiO} 2$ compositecatalysed click synthesis of 1,2,3-triazoles in water media. New J. Chem. 2014, 38, 1410-1417.

(46) Lipshutz, B. H.; Taft, B. R. Heterogeneous Copper-in-CharcoalCatalyzed Click Chemistry. Angew. Chem., Int. Ed. 2006, 45 (48), $8235-8238$.

(47) Sharghi, H.; Khalifeh, R.; Doroodmand, M. M. Copper Nanoparticles on Charcoal for Multicomponent Catalytic Synthesis of 1,2,3-Triazole Derivatives from Benzyl Halides or Alkyl Halides, Terminal Alkynes and Sodium Azide in Water as a "Green" Solvent. Adv. Synth. Catal. 2009, 351 (1-2), 207-218.

(48) Alonso, F.; Moglie, Y.; Radivoy, G.; Yus, M. Multicomponent Synthesis of 1,2,3-Triazoles in Water Catalyzed by Copper Nanoparticles on Activated Carbon. Adv. Synth. Catal. 2010, 352 (18), $3208-3214$

(49) Kale, S.; Kahandal, S.; Disale, S.; Jayaram, R. Conventional and microwave-assisted multicomponent reaction of alkyne, halide and sodium azide catalyzed by copper apatite as heterogeneous base and catalyst in water. Curr. Chem. Lett. 2012, 1, 69-80.

(50) Nador, F.; Volpe, M. A.; Alonso, F.; Feldhoff, A.; Kirschning, A.; Radivoy, G. Copper nanoparticles supported on silica coated maghemite as versatile, magnetically recoverable and reusable catalyst for alkyne coupling and cycloaddition reactions. Appl. Catal., A 2013, 455, 39-45.

(51) Baghbanzadeh, M.; Carbone, L.; Cozzoli, P. D.; Kappe, C. O. Microwave-Assisted Synthesis of Colloidal Inorganic Nanocrystals. Angew. Chem., Int. Ed. 2011, 50 (48), 11312-11359.

(52) Kruk, M.; Jaroniec, M. Gas Adsorption Characterization of Ordered Organic-Inorganic Nanocomposite Materials. Chem. Mater. 2001, 13 (10), 3169-3183.

(53) Roziere, J.; Brandhorst, M.; Dutartre, R.; Jacquin, M.; Jones, D. J.; Vitse, P.; Zajac, J. Effect of surfactant type, substitution by aluminium and additives on direct liquid crystal templated monolithic silica. J. Mater. Chem. 2001, 11 (12), 3264-3275.

(54) Tompsett, G. A.; Krogh, L.; Griffin, D. W.; Conner, W. C. Hysteresis and Scanning Behavior of Mesoporous Molecular Sieves. Langmuir 2005, 21 (18), 8214-8225.

(55) Gregg, S. J.; Sing, K. S. W. Adsorption, Surface Area, and Porosity, Second ed.; Academic Press: London, 1982.

(56) Chmielarz, L.; Kuśtrowski, P.; Dziembaj, R.; Cool, P.; Vansant, E. F. Catalytic performance of various mesoporous silicas modified with copper or iron oxides introduced by different ways in the selective reduction of NO by ammonia. Appl. Catal., B 2006, 62 (3-4), 369380.

(57) Trejda, M.; Tuel, A.; Kujawa, J.; Kilos, B.; Ziolek, M. Niobium rich SBA-15 materials - preparation, characterisation and catalytic activity. Microporous Mesoporous Mater. 2008, 110 (2-3), 271-278.

(58) Lee, C.-T.; Huang, S.; Lipshutz, B. H. Copper-in-CharcoalCatalyzed, Tandem One-Pot Diazo Transfer-Click Reactions. Adv. Synth. Catal. 2009, 351 (18), 3139-3142.

(59) Alvarez, S. G.; Alvarez, M. T. A practical procedure for the synthesis of alkyl azides at ambient temperature in dimethyl sulfoxide in high purity and yield. Synthesis 1997, 1997 (4), 413-414.

(60) Veerakumar, P.; Velayudham, M.; Lu, K.-L.; Rajagopal, S. Highly dispersed silica-supported nanocopper as an efficient heterogeneous catalyst: application in the synthesis of 1, 2, 3-triazoles and thioethers. Catal. Sci. Technol. 2011, 1 (8), 1512-1525.

(61) Himo, F.; Lovell, T.; Hilgraf, R.; Rostovtsev, V. V.; Noodleman, L.; Sharpless, K. B.; Fokin, V. V. Copper(I)-Catalyzed Synthesis of Azoles. DFT Study Predicts Unprecedented Reactivity and Intermediates. J. Am. Chem. Soc. 2005, 127 (1), 210-216.

(62) Siemsen, P.; Livingston, R. C.; Diederich, F. Acetylenic coupling: a powerful tool in molecular construction. Angew. Chem., Int. Ed. 2000, 39 (15), 2632-2657.

(63) Ellanki, A. R.; Islam, A.; Rama, V. S.; Pulipati, R. P.; Rambabu, D.; Rama Krishna, G.; Malla Reddy, C.; Mukkanti, K.; Vanaja, G. R.; 
Kalle, A. M.; Shiva Kumar, K.; Pal, M. Solvent effect on coppercatalyzed azide-alkyne cycloaddition (CuAAC): Synthesis of novel triazolyl substituted quinolines as potential anticancer agents. Bioorg. Med. Chem. Lett. 2012, 22 (10), 3455-3459.

(64) Glasnov, T. N.; Kappe, C. O. Microwave-Assisted Click Chemistry for the Preparation of 3- and 4-Triazolyl-2(1H)-quinolones as Potential Fluorescent Probes. QSAR Comb. Sci. 2007, 26 (11-12), $1261-1265$. 